\title{
Reheating constraints on the inflaton and dark matter: Swampland conjecture
}

\author{
Md Riajul Haque* and Debaprasad Maity ${ }^{\dagger}$ \\ Department of Physics, Indian Institute of Technology Guwahati, Guwahati, Assam 781039, India
}

(Received 2 March 2019; published 29 May 2019)

\begin{abstract}
In this paper, we studied the phenomenological consequences of recently proposed swampland conjecture on the inflationary models through constraints on reheating. If dark matter is assumed to be produced during reheating, the conjecture will provide further constraints on the dark matter parameter space through its current relic abundance. As has been pointed out already and also analyzed in our present paper, any successful inflationary scenario is in clear tension with the aforementioned conjecture in its current form. However considering the swampland parameters to be free and constrained by the inflationary observables, we studied in detail its consequence on the reheating and dark matter phenomenology. We point out the connection between swampland conjecture and the scalar spectral index $n_{s}$ by PLANCK within current $2 \sigma$ range, and associated constraints imposed on the reheating temperature and the dark matter annihilation cross section.
\end{abstract}

DOI: 10.1103/PhysRevD.99.103534

\section{INTRODUCTION}

An effective field theory framework has been the subject of intensive investigation for its universal appeal to diverse problems in physics. From large scale to small scale, wherever there exists a hierarchy of scales in the problem, it proves to be a unique and logical tool to understand the low scale properties by integrating out the high scale modes supplemented with a finite number of scale-dependent free parameters. However, the procedure suggests the existence of theory at a high energy scale, which is in general difficult to define. Therefore, the usual approach is to construct the low energy theory order by order in terms of low energy modes based on some underlying symmetry principle which is assumed to be the full theory property. A natural question then one can ask is whether all possible effective field theory so constructed can have its ultraviolet completion. This is a very difficult question to answer. String theory has been proven to be a fantastic playing field in this regard. This is the only theory, we know, which is at least an ultraviolet complete theory of gravity.

Recently motivated by this question and taking the help of various string theory constructions, a number of attempts have been made to put some constraints on the effective

\footnotetext{
riaju176121018@iitg.ac.in

debu@iitg.ac.in
}

Published by the American Physical Society under the terms of the Creative Commons Attribution 4.0 International license. Further distribution of this work must maintain attribution to the author(s) and the published article's title, journal citation, and DOI. Funded by SCOAP ${ }^{3}$. theory which will have consistent UV completion. One such proposal is the swampland conjecture [1], which has recently gained interest in the literature. The conjecture says that a low energy effective theory of scalar field minimally coupled with gravity must satisfy the following universal bound on its form of the potential:

$$
\frac{|\nabla V|}{V} \geq \frac{c}{M_{p}}
$$

where $c$ is a dimensionless constant with the magnitude of order unity and $M_{p}$ is the Planck mass. However, there exists a refined version of the aforementioned swampland conjecture stated in [1] which is expressed as

$$
\frac{|\nabla V|}{V} \geq \frac{c}{M_{p}} \quad \text { or } \quad \min \left(\nabla_{i} \nabla_{j} V\right) \leq-\frac{c^{\prime}}{M_{p}^{2}} .
$$

This is a weaker condition on the possible form of the potential. $c^{\prime}$ is another universal constant of order unity, and $\min \left(\nabla_{i} \nabla_{j} V\right)$ is the minimum eigenvalue of the Hessian of $\nabla_{i} \nabla_{j} V$ matrix in an orthogonal frame.

Another interesting criterion which has long been considered already in the context of inflation is the amount of field excursion $\delta \phi$ with regard to the famous Lyth bound [2]. It is pointed out that effective field theory description will be valid at the two different points in the field space provided the difference satisfies the following condition:

$$
\frac{\delta \phi}{M_{p}} \leq \Delta
$$


where $\Delta$ is the order one constant. This conjecture is known as swampland distance conjecture $[3,4]$. Our goal of this paper would be to discuss the implications of those conjectures specifically during the reheating period.

A large number of studies have been performed over the years to understand more about the theoretical understanding of the swampland conjecture [5-53]. However, it would be important to mention the interesting debates going on in the literature on the existence of the de Sitter vacuum in string theory $[54,55]$. From the phenomenological point of view, the implication of this conjecture has been widely studied in the context of cosmology [56-62]. However, few studies have been done in the context of reheating, as it is the phase which sets in the initial condition for subsequent evolution of our Universe after the inflation. In this paper we will be specifically focusing on this particular phase. Starting from inflation to dark energy, the scalar field is ubiquitous and therefore, the conjecture can naturally put constraints on the model building. More interestingly, the hope is that the inflationary and dark energy observation may shed light on UV physics through these conjectures.

As mentioned above, in this paper, we will consider inflationary models with a specific interest on the reheating dynamics. We ask the following question: How does the swampland conjecture put constraints in the reheating dynamics and the dark matter phenomenology? In our analysis, we will consider $\left(c, c^{\prime}\right.$ and $\left.\Delta\right)$ as free parameters. Taking constraints on those parameters from the inflationary dynamics, we will further study the reheating phase.

In the subsequent section we first briefly review the basic equations describing the constraints on reheating and consequently on the dark matter parameters considering the cosmic microwave background (CMB) anisotropy and the current dark matter abundance. We take four different types of inflationary model potentials and describe how the swampland conjecture restricts the reheating and the dark matter parameter space through the inflationary observables.

\section{REHEATING AND DARK MATTER: METHODOLOGY}

Reheating is the phase which connects the inflation and standard big-bang evolution of our Universe through decay of inflaton. This phase has also been proved to play an important role in the dark matter phenomenology. Even though inflation is severely constrained by a large number of cosmological observations, the reheating phase is generally unconstrained. The phase is parametrized by two important physical quantities called reheating temperature $T_{r e}$ and $e$-folding number $N_{r e}$. To go beyond we further incorporate the production of dark matter during this phase. During reheating inflaton decays to radiation and then it annihilates to dark matter such that the process during reheating gives us correct relic abundance. As emphasized before, given the observational constraints on the inflationary dynamics, our goal of this paper would be to constrain the reheating and dark matter parameter space through the inflationary parameters considering the swampland conjecture.

For simplicity, we first follow the usual reheating constraint analysis [63] where the reheating parameters are calculated assuming the instantaneous conversion of inflaton energy into radiation at the instant of reheating. The evolution during reheating is parametrized by an effective constant equation of state $w_{\text {re }}$. Following the aforementioned assumptions and considering a particular inflation model, one can easily compute the reheating temperature to be

$$
T_{r e}=\left(\frac{43}{11 g_{r e}}\right)^{\frac{1}{3}}\left(\frac{a_{0} T_{0}}{k}\right) H_{k} e^{-N_{k}} e^{-N_{r e}},
$$

where $g_{r e}$ is the effective number of relativistic degrees of freedom at the instant of reheating. $\left(T_{0}=2.725 \mathrm{~K}, a_{0}\right)$ are the CMB temperature, and the cosmological scale factor at the present time respectively. The number of reheating $e$-folding number during reheating can be expressed as [64]

$$
\begin{aligned}
N_{r e}= & \frac{4}{\left(1-3 \omega_{r e}\right)}\left[-\frac{1}{4} \ln \left(\frac{45}{\pi^{2} g_{r e}}\right)-\frac{1}{3} \ln \left(\frac{11 g_{r e}}{43}\right)\right. \\
& \left.-\ln \left(\frac{k}{a_{0} T_{0}}\right)-\ln \left(\frac{V_{\text {end }}^{1 / 4}}{H_{k}}\right)-N_{k}\right] .
\end{aligned}
$$

Flrom the above equation, we can clearly see the appearance of inflationary parameters which are constrained by the swampland conjecture. Therefore, indirect constraints can be imposed on the reheating parameter space. We will be considering some simple canonical scalar field models of inflation, for which Hubble constant $H_{k}$ and the inflationary $e$-folding number $N_{k}$ are defined as

$H_{k}=\frac{\pi M_{p} \sqrt{r_{k} A_{s}\left(n_{s}^{k}\right)}}{\sqrt{2}} ; \quad N_{k}=\int_{\phi_{k}}^{\phi_{\mathrm{end}}} \frac{|d \phi|}{\sqrt{2 \epsilon_{V}} M_{p}}$.

The nontrivial dependence of reheating parameters on the scalar spectral index, $n_{s}^{k}$, and the tensor to scalar ratio, $r_{k}$, through the power spectrum of inflaton fluctuation, $A_{s}\left(n_{s}^{k}\right)$, will play the key role in establishing the connection among the swampland parameters and the reheating parameters. The aforementioned well-known inflationary parameters,

$$
n_{s}^{k}=1-6 \epsilon_{V}+2 \eta_{V} ; \quad r_{k}=16 \epsilon_{V},
$$

in turn depend on the slow roll parameters related to the inflaton potential $V(\phi)$, which can now be constrained by the swampland conjecture,

$$
\epsilon_{V}=\frac{M_{p}^{2}}{2}\left(\frac{V^{\prime}}{V}\right)^{2} ; \quad\left|\eta_{V}\right|=M_{p}^{2} \frac{\left|V^{\prime \prime}\right|}{V} .
$$


Most importantly all of the above quantities are defined at a particular cosmological scale $k$. For CMB, we consider the pivot scale of PLANCK $k / a_{0}=0.002 \mathrm{Mpc}^{-1}$. The end of inflation set the initial condition for the reheating dynamics. Therefore, dynamics will be mostly controlled by $V\left(\phi_{\text {end }}\right)$, where $\phi_{\text {end }}$ is the inflaton field value at the end of inflation and follows from the equation $\epsilon_{V}\left(\phi_{\text {end }}\right)=1$.

In the discussion so far we have not considered explicit decay of inflaton. However to shed light on the dark matter phenomenology we consider the perturbative reheating process where inflation decays to radiation and then radiation to dark matter [65]. For this system, we will have three parameters, the inflaton decay constant $\Gamma_{\phi}$, the thermal average of dark matter annihilation cross section $\langle\sigma v\rangle$, and the dark matter mass $M_{X}$. In the perturbative reheating process, the dynamics of the inflaton energy density $\left(\rho_{\phi}\right)$, the radiation energy density $\left(\rho_{R}\right)$ and dark matter particle number density $\left(n_{X}\right)$ are modeled by the following homogeneous Boltzmann equations [65]:

$$
\begin{gathered}
\Phi^{\prime}=-c_{1} \frac{A^{1 / 2} \Phi}{\sqrt{\Phi+R / A+X\left\langle E_{X}\right\rangle / m_{\phi}}} \\
R^{\prime}=c_{1} \frac{A^{3 / 2} \Phi}{\sqrt{\Phi+R / A+X\left\langle E_{X}\right\rangle / m_{\phi}}} \\
+c_{2} \frac{A^{-3 / 2}\langle\sigma v\rangle 2\left\langle E_{X}\right\rangle M_{p l}}{\sqrt{\Phi+R / A+X\left\langle E_{X}\right\rangle / m_{\phi}}}\left(X^{2}-X_{e q}^{2}\right) ; \\
X^{\prime}=-c_{2} \frac{A^{-5 / 2}\langle\sigma v\rangle M_{p l} m_{\phi}}{\sqrt{\Phi+R / A+X\left\langle E_{X}\right\rangle / m_{\phi}}}\left(X^{2}-X_{e q}^{2}\right),
\end{gathered}
$$

where, for numerical purpose, the dimensionless variables are defined as

$$
\Phi \equiv \frac{\rho_{\phi} a^{3}}{m_{\phi}} ; \quad R \equiv \rho_{R} a^{4} ; \quad X \equiv n_{X} a^{3} .
$$

We also rescale the cosmological scale factor by $A=\frac{a}{a_{\text {end }}}$, where $a_{\text {end }}$ is the scale factor at the end of inflation. Prime ' represents the derivative with respect to $A$. The inflation mass is $m_{\phi}$. The average energy of the dark matter particle is expressed as $\left\langle E_{X}\right\rangle=\frac{\rho_{X}}{n_{X}} \simeq \sqrt{M_{X}^{2}+9 T^{2}}$ [66]. The equilibrium number density of dark matter particle of mass $M_{X}$ can be expressed in terms of modified Bessel function of the second kind [66],

$$
n_{X}^{e q}=\frac{g T^{3}}{2 \pi^{2}}\left(\frac{M_{X}}{T}\right)^{2} K_{2}\left(\frac{M_{X}}{T}\right) .
$$

The constants $c_{1}$ and $c_{2}$ are defined as

$$
c_{1}=\frac{\sqrt{\frac{3}{8 \pi}} M_{p l} \Gamma_{\phi}}{m_{\phi}^{2}} ; \quad c_{2}=\sqrt{\frac{3}{8 \pi}} .
$$

The initial conditions to solve the Boltzmann equations are set at the end of inflation to be

$$
\Phi(1)=\frac{3}{2} \frac{V\left(\phi_{\mathrm{end}}\right)}{m_{\phi}^{4}} ; \quad R(1)=X(1)=0 .
$$

In this process, we will define reheating temperature $\left(T_{r e}\right)$ from the radiation temperature $\left(T_{\mathrm{rad}}\right)$, at the instant of maximum energy transfer from inflaton to radiation for $H(t)=\Gamma_{\phi}$ :

$$
T_{r e}=T_{\mathrm{rad}}^{\mathrm{end}}=\left(\frac{30}{\pi^{2} g_{*}(T)}\right)^{1 / 4} \rho_{R}\left(\Gamma_{\phi}, N_{r e}, n_{s}^{k}\right)^{1 / 4} .
$$

Combining this equation with Eq. (4) we can establish one to one correspondence between $T_{r e}$ and $\Gamma_{\phi}$ supplemented with the following condition for reheating:

$$
\begin{aligned}
H^{2} & =\dot{N}_{r e} \\
& =\frac{\rho_{\phi}\left(\Gamma_{\phi}, N_{r e}, n_{s}^{k}\right)+\rho_{R}\left(\Gamma_{\phi}, N_{r e}, n_{s}^{k}\right)+\rho_{X}\left(\Gamma_{\phi}, N_{r e}, n_{s}^{k}\right)}{3 M_{p}^{2}} \\
& =\Gamma_{\phi}^{2}
\end{aligned}
$$

While solving the above Boltzmann equation the dark matter abundance parametrized by $\Omega_{X}$, and expressed in terms of radiation abundance $\Omega_{R}\left(\Omega_{R} h^{2}=4.3 \times 10^{-5}\right)$, is set to the following observed value:

$$
\Omega_{X} h^{2}=\left\langle E_{X}\right\rangle \frac{X\left(T_{F}\right) T_{F} A_{F}}{R\left(T_{F}\right) T_{n o w} m_{\phi}} \Omega_{R} h^{2}=0.12
$$

where $T_{F}$ is the temperature at a very late time when comoving dark matter, as well as radiation density, became constant. The present value of dark matter abundance imposes a constraint on the dark matter parameter space $\left(M_{X},\langle\sigma v\rangle\right)$ through the CMB anisotropies and the swampland conjecture.

Therefore, inflationary dynamics, and considering the CMB temperature anisotropy, we will be able to constrain reheating as well as dark matter parameter space through the swampland conjecture. With all the ingredients discussed so far, and considering the condition of the refined swampland conjecture Eq. (2) and swampland distance conjecture Eq. (3), we can figure out the allowed region of $\left(c, c^{\prime}\right.$ and $\left.\Delta\right)$ with respect to inflation parameters $\left(n_{s}^{k}, r_{k}\right)$. The value of $n_{s}^{k}$ will be considered to be constrained by the PLANCK $2 \sigma$ region in the $\left(n_{s}^{k}, r\right)$ plane. With this consideration, a particular value of $c$ can be shown to provide the maximum allowed value of $n_{s}^{k}$ which in turn imposes a 
restriction not only on the maximum value of the reheating temperature $\left(T_{r e}^{\max }\right)$ but also on the dark matter parameter space allowed by current dark matter abundance. We also, additionally, show that a particular value of $\Delta$ will put a new restriction on the maximum values of reheating temperature and the dark matter parameter space. Similarly, the maximum allowed value of reheating temperature, which is associated with a particular value of the spectral index, should also impose constraints on $c$ and $\Delta$. We show the resulting constraints on $c$ and $c^{\prime}$ for the maximum value of the scalar spectral index $\left(n_{s}^{\max }\right)$ and the minimum value of the scalar spectral index $\left(n_{s}^{\mathrm{min}}\right)$. In the following discussions, we consider various inflation models and discuss the important results of our analysis. In all cases, we consider two different effective equations of state parameter for reheating, $\omega_{r e}=0$ and $\omega_{r e}=\frac{1}{6}$.

\section{A. Chaotic inflation [67]}

For the usual chaotic ination model the potential looks like

$$
V(\phi)=\frac{1}{2} m^{4-n} \phi^{n},
$$

where $n=2,4,6, \ldots$. If we consider the absolute value of the field, $n=3,5, \ldots$ can also be included. We consider only $n=2$ for our numerical purpose. As mentioned earlier, the first condition of the refined swampland conjecture, $M_{p} V^{\prime} / V$, transforms into the following inequality:

$$
M_{p} \frac{V^{\prime}}{V}=M_{p} \frac{2}{\phi_{k}} \geq c,
$$

where the inflaton field value for a particular scale of interest $k$ can be written as

$$
\phi_{k}^{2}=\frac{1}{1-n_{s}^{k}}\left(3 M_{p}^{2} n^{2}-2 n(n-1) M_{p}^{2}\right) .
$$

After combining Eqs. (20) and (21), we see that a particular value of $c$ gives an upper bound on the value of scalar spectral index $n_{s}^{k}$. This constraint sets a maximum possible value of the reheating temperature $\left(T_{r e}^{\max }\right)$. Because of the implicit relation, the above constraint also sets a minimum possible value of dark matter scattering cross section $\langle\sigma v\rangle$ for a given dark matter mass. Similarly from the second condition of the conjecture, we find the following further constraint on the inflaton field value:

$$
M_{p}^{2} \frac{V^{\prime \prime}}{V}=\frac{2}{\phi_{k}^{2}} M_{p}^{2} \leq-c^{\prime} .
$$

Combing Eqs. (20), (21) and (22), we are able to find constraints on $c, c^{\prime}$. For $n=2$, the combined equation can be written as

$$
c^{2} c^{\prime 2} \leq \frac{1}{32}\left(1-n_{s}^{k}\right)^{3} .
$$

By using the above constraint relation along with any one of the swampland conjectures, we have the allowed region of the $\left(c, c^{\prime}\right)$ space as shown in Fig. 1.

Further using the swampland distance conjecture, one obtains the following inequality in terms of spectral index and inflationary $e$-folding number:

$$
\frac{\Delta \phi}{M_{p}}=\frac{r_{k}^{\frac{1}{2}} N_{k}}{2 \sqrt{2}}=\frac{\sqrt{\frac{n\left(1-n_{s}\right)}{2+n}}\left(4+n\left(1+n_{s}\right)\right)}{4\left(n_{s}-1\right)} \leq \Delta .
$$

Once we have the mathematical relations between the reheating parameters and the swampland parameters, in the following we discuss their implication and various constraints. For the chaotic model, there exists a maximum value of the scalar spectral index, such as for $n=2, n_{s}^{\max } \simeq$ 0.96726 and it remains same for different effective reheating equation of state $\omega_{r e}$ as can be inferred from the first plot of Fig. 1. Associated with this maximum possible temperature, we have a unique value of $c=c^{t \max } \simeq 0.128$. Most importantly, if we consider the lowest value of the reheating temperature to be at the time of $\mathrm{BBN}$, within the PLANCK limit, a minimum possible value of the spectral index $n_{s}^{\min } \simeq(0.95806,0.96388)$ can be identified for different values of $\omega_{r e}=\left(0, \frac{1}{6}\right)$, and consequently a maximum possible value of $c^{\max }=(0.1448,0.1344)$ arises. The most important result of our present analysis is for each value of $c$ between $\left(c^{\max }>c>c^{t \max }\right)$, there exists a maximum allowed value of reheating temperature $T_{r e}^{\max }(c)$ for a given equation of state. For example for $c=0.130$, the maximum allowed value of the reheating temperature turns out to be $T_{r e}^{\max }(0.13)=\left(8.4 \times 10^{12}, 3.7 \times 10^{9}\right) \mathrm{GeV}$ for $\omega_{r e}=\left(0, \frac{1}{6}\right)$ respectively. In the third plot of Fig. 1 we have shown the allowed region of the swampland parameter $c$ in terms of scalar spectral index $n_{s}$. Here we can see that the allowed region of $c$ starts decreasing with the increasing effective equation of state $\omega_{r e}$.

In the fourth plot of Fig. 1, we have plotted the annihilation cross section as a function of $n_{s}$ for different dark matter masses within the maximum and minimum values of the reheating temperature. For a given value of $c$, one can precisely predict the values of the annihilation cross section once dark matter mass is fixed. As an example given the value $c=0.130$, the current relic abundance fixes the dark matter annihilation cross section within $8.8 \times 10^{-19} \mathrm{GeV}^{-2}>\langle\sigma v\rangle>1.5 \times 10^{-39} \mathrm{GeV}^{-2}$ for $M_{X}=$ $10^{3} \mathrm{GeV}$ and $2.3 \times 10^{-19} \mathrm{GeV}^{-2}>\langle\sigma v\rangle>1.8 \times$ $10^{-42} \mathrm{GeV}^{-2}$ for $M_{X}=10^{6} \mathrm{GeV}$. Importantly considering the PLANCK observation, if we increase the value of $c$ towards $c^{\text {max }}$, this range of dark matter annihilation cross section will be further narrowed down.

It is a well-known fact that chaotic inflation requires super-Planckian field excursion in order to obtain the correct value of the scalar spectral index. Therefore, one would naturally expect the swampland parameter $\Delta$ related to distance conjecture to be larger than unity. However, as has been emphasized before, like $\left(c, c^{\prime}\right)$, we consider $\Delta$ as a 

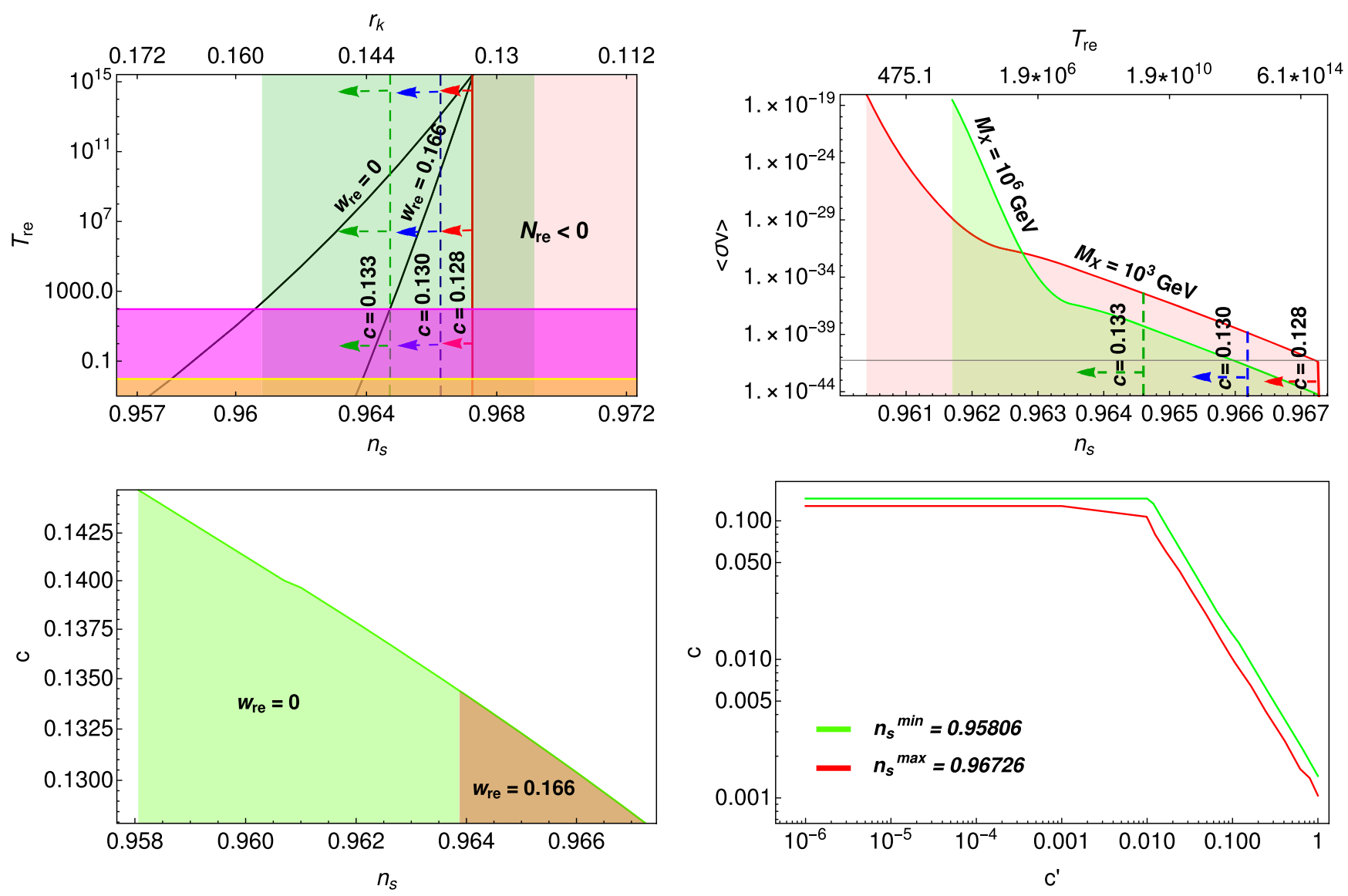

FIG. 1. We plot on the upper left side variation of the reheating temperature $\left(T_{r e}\right)$ as a function of the spectral index $\left(n_{s}\right)$ for two effective equations of state, $\omega_{r e}=0$ and $\omega_{r e}=\frac{1}{6}$. A red solid line indicates the maximum value of the spectral index $\left(n_{s}^{\max }\right)$ which corresponds to $c=0.128$. The intersection point of the temperature curves and the red solid line indicates the maximum value of the reheating temperature $\left(T_{r e}^{\max }\right)$. Temperature above the intersection point is unphysical as they correspond to $N_{r e}<0$ (light red region). The light green band indicates $1 \sigma$ range, $n_{s}=0.9649 \pm 0.0042$ (68\% C.L., Planck TT, TE, EE + low E + lensing) from Planck [68] and the dark pink region is below the electroweak scale $T_{e w} \sim 100 \mathrm{GeV}$. The dark yellow region below $10^{-2} \mathrm{GeV}$ would ruin the predictions of big bang nucleosynthesis (BBN). Different (shown in different color dashed lines) values of $c$ impose a further restriction on the maximum value of the reheating temperature. In the upper right side, we have plotted the contour of $\Omega_{X} h^{2}=0.12$ in the $n_{s}-\langle\sigma v\rangle$ plane for fixed dark matter masses. The shaded region below the contour line is the allowed parameter space, which is further constrained by different values of $c$ (shown in different colours). On the lower left side, we plot allowed values of $c$ as a function of $n_{s}$ within maximum and minimum values of the reheating temperature. The region under the green solid line is for $\omega_{r e}=0$ and the dark red region for $\omega_{r e}=\frac{1}{6}$. In the lower right side, we show the resulting constraints for $c$ and $c^{\prime}$ for maximum and minimum values of the spectral index with considering effective equation of state $\omega_{r e}=0$. Minimum and maximum values of the spectral index correspond to $T_{r e} \sim 10^{-2} \mathrm{GeV}$ and $N_{r e} \sim 0$ respectively. All four plots are for the chaotic inflation model with $n=2$.

free parameter and discuss its possible bounds and observational consequence on the dark matter phenomenology. As was the case for other swampland parameters, the maximum and minimum values of the spectral index restrict the allowed parameter space of minimum values of $\Delta=\Delta^{\mathrm{min}}$, to be within (6.83-7.75) as shown in Fig. 5. Therefore, given the implicit relation between $n_{s}$ and $T_{r e}$, a particular value of $\Delta$ within the aforementioned range corresponds to a maximum value of reheating temperature and consequently a minimum value of the dark matter annihilation cross section. For example, if we take $\Delta=7.6$, our analysis gives the maximum allowed values of reheating temperature, $\quad T_{r e}^{\max }=\left(2.97 \times 10^{12}, 3.23 \times 10^{8}\right) \mathrm{GeV}$ for $\omega_{r e}=\left(0, \frac{1}{6}\right)$ and the dark matter annihilation cross section within $8.8 \times 10^{-19} \mathrm{GeV}^{-2}>\langle\sigma v\rangle>3.7 \times 10^{-39} \mathrm{GeV}^{-2}$ for $M_{X}=10^{3} \mathrm{GeV}$ and $2.3 \times 10^{-19} \mathrm{GeV}^{-2}>\langle\sigma v\rangle>$ $3.7 \times 10^{-42} \mathrm{GeV}^{-2}$ for $M_{X}=10^{6} \mathrm{GeV}$. Importantly unlike $c$, with increasing $\Delta$ within the allowed range, the range of cross section becomes wider.

\section{B. Natural inflation [69]}

The inflationary potential in this model is given by

$$
V(\phi)=\Lambda^{4}\left[1-\cos \left(\frac{\phi}{f}\right)\right]
$$


where $\Lambda$ is the height of the potential setting the inflationary energy scale, and $f$ is the width of the potential, known as the axion decay constant. The CMB normalization fixes the overall scale of the inflation $\Lambda$. Therefore, by tuning the value of the axion decay constant $f$ we can fit this model with observation. We have chosen two sample values of axion decay constant $f=\left(10 M_{p}, 50 M_{p}\right)$ and those values of decay constant are marginally consistent with Planck data. The maximum reheating temperature $T_{r e}^{\max } \sim 10^{15} \mathrm{GeV}$ for different values of $f$ and $\omega_{r e}$, arising due to instant reheating $\left(N_{r e} \sim 0\right)$ sets the maximum possible value of scalar spectral index $n_{s}^{\max } \simeq(0.96617,0.96726)$ for $f=\left(10 M_{p}, 50 M_{p}\right)$ respectively. However as already discussed for the chaotic inflation, the maximum possible value of $c=c^{\max }$ arises from the minimum possible values of spectral index which corresponds to a minimum value of the reheating temperature. The minimum values of the spectral index $n_{s}^{\text {min }} \simeq$ $(0.95717,0.9629)$ for $\left(\omega_{r e}, f\right)=\left(\left(0,10 M_{p}\right),\left(\frac{1}{6}, 10 M_{p}\right)\right)$ and $n_{s}^{\min } \simeq(0.9581,0.9639)$ for $\left(\omega_{r e}, f\right)=\left(\left(0,50 M_{p}\right)\right.$, $\left.\left(\frac{1}{6}, 50 M_{p}\right)\right)$. With these ingredients the constraints on $c$ will be obtained from the refined swampland conjecture [Eqs. (2) and (22)] which transforms into the following inequalities for the axion inflaton:

$$
\begin{gathered}
M_{p} \frac{V^{\prime}}{V}=\frac{M_{p}}{f} \cot \left(\frac{\phi}{2 f}\right)=\frac{M_{p}}{f} \sqrt{\frac{f^{2}}{2 M_{p}^{2}}\left(1-n_{s}\right)-\frac{1}{2}} \geq c \\
M_{p}^{2} \frac{V^{\prime \prime}}{V}=\left(\frac{f^{2}\left(1-n_{s}\right)-3 M_{p}^{2}}{4 f^{2}}\right) \leq-c^{\prime}
\end{gathered}
$$

Additionally, from the swampland distance conjecture Eq. (5) we get the following inequality in terms of reheating parameters:

$$
\frac{\Delta \phi}{M_{p}}=\frac{r_{k}^{\frac{1}{2}} N_{k}}{2 \sqrt{2}} \leq \Delta,
$$

where

$$
\begin{aligned}
N_{k} & =\frac{f^{2}}{M_{p}^{2}} \ln \left(\frac{2 f^{2}\left(f^{2}\left(1-n_{s}\right)+M_{p}^{2}\right)}{\left(2 f^{2}+M_{p}^{2}\right)\left(f^{2}\left(1-n_{s}\right)-M_{p}^{2}\right)}\right), \\
r_{k} & =4\left(\frac{f^{2}\left(1-n_{s}\right)-M_{p}^{2}}{f^{2}}\right) .
\end{aligned}
$$

It is important to note that, at the instant of instantaneous reheating $\left(N_{r e} \sim 0\right)$, the associated value of $c\left(c^{t \max }\right)$ is independent of the reheating equation of state but dependent on the axion decay constant $f$, such as $c^{t \max }=$ $(0.109,0.127)$ for $f=(10,50) M_{p}$ for both values of equation of state. As was the case for chaotic inflation, Eq. (26) which constrains the value of spectral index as $n_{s} \leq 1-\left(2 c^{2}+\frac{M_{p}^{2}}{f^{2}}\right)$ will directly restrict the possible value of the reheating temperature for a given value of $c>c^{t \max }$. For fixed values of $c$ (greater than $c^{t \max }$ ) and $f$, maximum allowed values of reheating temperature are different for different equations of state $\omega_{r e}$. Considering $(f, c)=$ $(50,0.1) M_{p}$, the maximum allowed values of reheating temperature turn out to be $T_{r e}^{\max }=\left(1.1 \times 10^{12}\right.$, $\left.3.1 \times 10^{7}\right) \mathrm{GeV}$ for $\omega_{r e}=(0,1 / 6)$ respectively. Similarly maximum and minimum values of reheating temperature from observation impose a restriction on the allowed values of $c$, which we have shown in the ( $c$ vs $n_{s}$ ) plot in Fig. 2. If we further consider the refined conjecture, combining two equations (26) and (27) one finds

$c^{2} c^{\prime 2} \leq \frac{1}{32}\left(\frac{f^{2}\left(1-n_{s}\right)-3 M_{p}^{2}}{f^{3}}\right)^{2}\left(f^{2}\left(1-n_{s}\right)-M_{p}^{2}\right)$.

Consequently the resulting constraints in $\left(c, c^{\prime}\right)$ space have been plotted in the last plot of Fig. 2, where the upper limit on $c$ has been derived from the PLANCK constraints.

Now we turn to understand the dark matter parameter space, specifically how the parameter $c$ constrains the dark matter annihilation cross section, $\langle\sigma v\rangle$, for different scalar spectral index or reheating temperature. Similar to the observation made for the chaotic inflation model, for a fixed value of $c=0.13$ and $f=50 M_{p}$, the annihilation cross section turns out to be constrained within $7.9 \times 10^{-19} \mathrm{GeV}^{-2}>\langle\sigma v\rangle>1.4 \times 10^{-38} \mathrm{GeV}^{-2}$, considering $M_{X}=10^{3} \mathrm{GeV}$ and $2 \times 10^{-19} \mathrm{GeV}^{-2}>\langle\sigma v\rangle>$ $1.8 \times 10^{-41} \mathrm{GeV}^{-2}$ for $M_{X}=10^{6} \mathrm{GeV}$. Furthermore, the lower limit of the annihilation cross section $\left(\langle\sigma v\rangle^{\text {lower }}\right)$ assumes higher value as one increases the values of $c$. All these feature are similar to the one we discussed for the chaotic inflation case.

As we noticed in the chaotic inflation model, for natural inflation also one has super-Planckian field excursion. Therefore, the swampland parameter $\Delta$ will always be larger than unity for the observed scalar spectral index within the PLANCK $2 \sigma$ range. Regarding constraining the value of $\Delta$, assuming a particular value of axion decay constant $f=(10,50) M_{p}$, we find $\Delta^{\mathrm{min}}$ within the following range [(6.03-6.60),(6.80-7.70)] respectively. Consequently, those minimum values set a maximum possible value of the reheating temperature and a minimum value of the dark matter annihilation cross section. For example, if we assume $\Delta=6.58$, we have $T_{r e}^{\max }=\left(9.8 \times 10^{14}, 2.5 \times 10^{14}\right) \mathrm{GeV}$ for $\omega_{r e}=(0,1 / 6)$ and consequently the dark matter annihilation cross section becomes restricted within $3.6 \times 10^{-19} \mathrm{GeV}^{-2}>\langle\sigma v\rangle>1.1 \times 10^{-41} \mathrm{GeV}^{-2}$ for $M_{X}=$ $10^{3} \mathrm{GeV}$ and $4.3 \times 10^{-20} \mathrm{GeV}^{-2}>\langle\sigma v\rangle>1.2 \times$ $10^{-44} \mathrm{GeV}^{-2}$ for $M_{X}=10^{6} \mathrm{GeV}$ with axion decay constant $f=10 M_{p}$. 

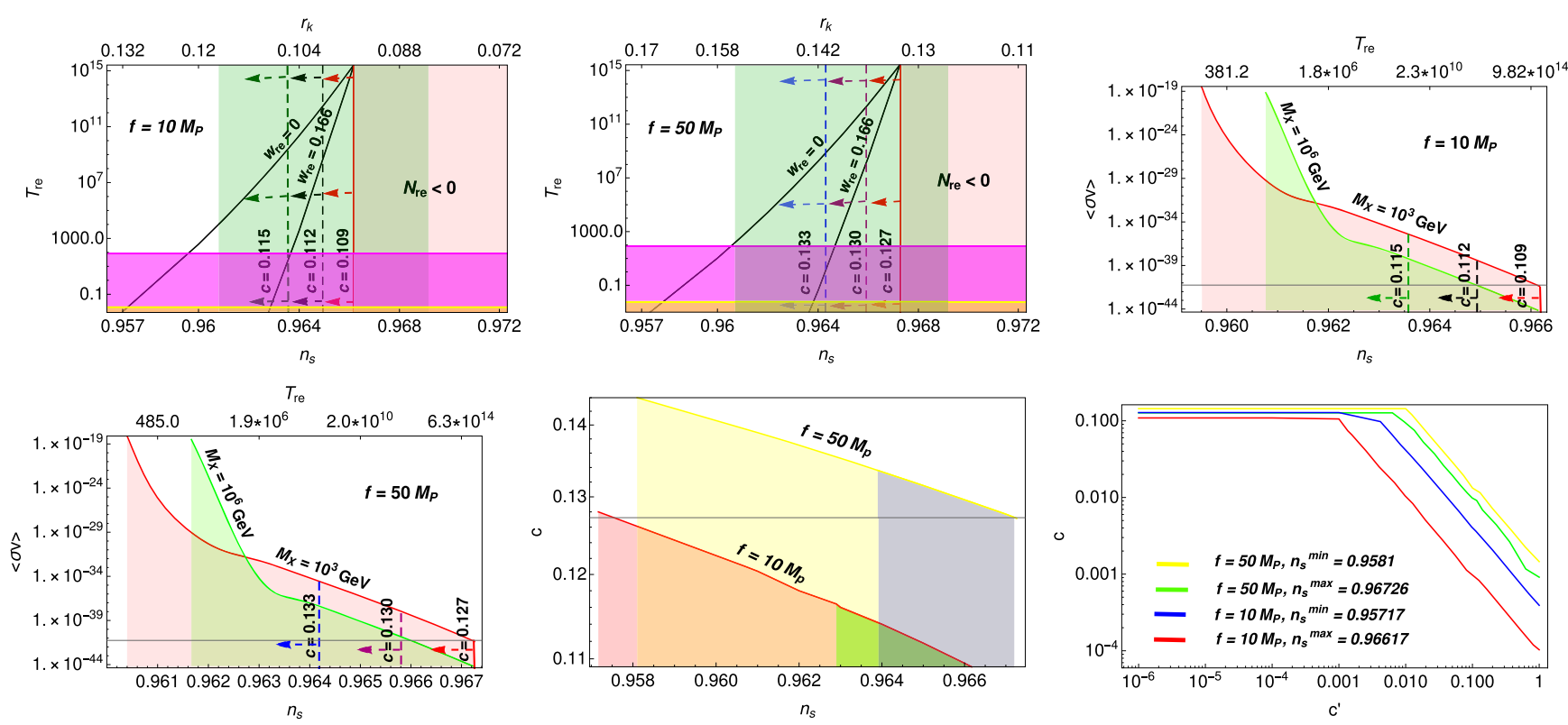

FIG. 2. All plots are the same as in Fig. 1. The only difference is that here we have plotted for the natural inflation model for $f=\left(10 M_{p}, 50 M_{p}\right)$. In the lower middle plot, the region under the yellow solid line is for $\left(\omega_{r e}, f\right)=\left(0,50 M_{p}\right)$ and the dark blue region is for $\left(\omega_{r e}, f\right)=\left(\frac{1}{6}, 50 M_{p}\right)$. In the same plot, the region under the red solid line is for $\left(\omega_{r e}, f\right)=\left(0,10 M_{p}\right)$ and the dark green region is for $\left(\omega_{r e}, f\right)=\left(\frac{1}{6}, 10 M_{p}\right)$.

\section{C. $\alpha$-attractor model [70]}

In this section, we will consider a class of theoretical models which unifies a large number of inflationary scenarios parametrized by $\alpha$, known as $\alpha$-attractor [70]. The conformal property of this class of models leads to a universal prediction for the inflationary observables. In the canonical framework the potential of this model is expressed as

$$
V(\phi)=\Lambda^{4}\left[1-e^{-\sqrt{\frac{2}{3 \alpha M_{p}}}}\right]^{2 n} .
$$

The mass scale $\Lambda$ which sets the energy scale of inflation can naturally be fixed from the CMB power spectrum. The parameter $\alpha$ determines the shape of the canonically normalized inflaton potential near its minimum. This model includes the Starobinsky model for $n=1$ and $\alpha=1$. In our present analysis we consider two cases for $\alpha=(1,100)$ with $n=1$ and compare their outcomes. For this choice of parameters both maximum and minimum values of the scalar spectral index correspond to the respective maximum and minimum values of the reheating temperature defined within the $2 \sigma$ range of PLANCK. The maximum value of the spectral index turns out to be $n_{s}^{\max } \simeq(0.96717,0.9702)$ for $\alpha=1$ and 100 respectively. Once again the value of the $n_{s}^{\max }$ remains the same for different equation of state $\omega_{r e}$ when $\alpha$ remains fixed. From the predictions of big bang nucleosynthesis (BBN), the minimum values of spectral index $n_{s}^{\min }$ are different for a different $\omega_{r e}$ and $\alpha$, such as $n_{s}^{\min } \simeq$ $(0.9579,0.96375)$ for $\left(\omega_{r e}, \alpha\right)=\left((0,1),\left(\frac{1}{6}, 1\right)\right)$ and $n_{s}^{\min } \simeq$ $(0.9616,0.96704)$ for $\left(\omega_{r e}, \alpha\right)=\left((0,100),\left(\frac{1}{6}, 100\right)\right)$.
In order to study the constraints on the swampland parameters, we consider the following inequalities as a function of inflationary model parameter in terms of $\left(n_{s}\right)$,

$$
M_{p} \frac{V^{\prime}}{V}=\frac{2 n \sqrt{\frac{2}{3 \alpha}} e^{-\sqrt{\frac{2}{3 \alpha}} \frac{\phi}{M_{p}}}}{1-e^{-\sqrt{\frac{2}{3 \alpha}} \frac{\phi}{M_{p}}}} \geq c,
$$

or

$$
M_{p}^{2} \frac{V^{\prime \prime}}{V}=\frac{4 n}{3 \alpha} e^{-\sqrt{\frac{2}{3 \alpha M_{p}}}}\left(\frac{2 n e^{-\sqrt{\frac{2}{3 \alpha M_{p}}}}-1}{\left(1-e^{-\sqrt{\frac{2}{3 \alpha M_{p}}}}\right)^{2}}\right) \leq-c^{\prime},
$$

where $\phi$ can be written in terms of spectral index $\left(n_{s}\right)$ as [71]

$$
\begin{aligned}
\phi= & \sqrt{\frac{3 \alpha}{2}} M_{p} \\
& \times \ln \left(1+\frac{4 n+\sqrt{16 n^{2}+24 \alpha n(1+n)\left(1-n_{s}\right)}}{3 \alpha\left(1-n_{s}\right)}\right) .
\end{aligned}
$$

Further, the distance conjecture transforms into the following form:

$$
\frac{\Delta \phi}{M_{p}}=\frac{r_{k}^{\frac{1}{2}} N_{k}}{2 \sqrt{2}} \leq \Delta
$$



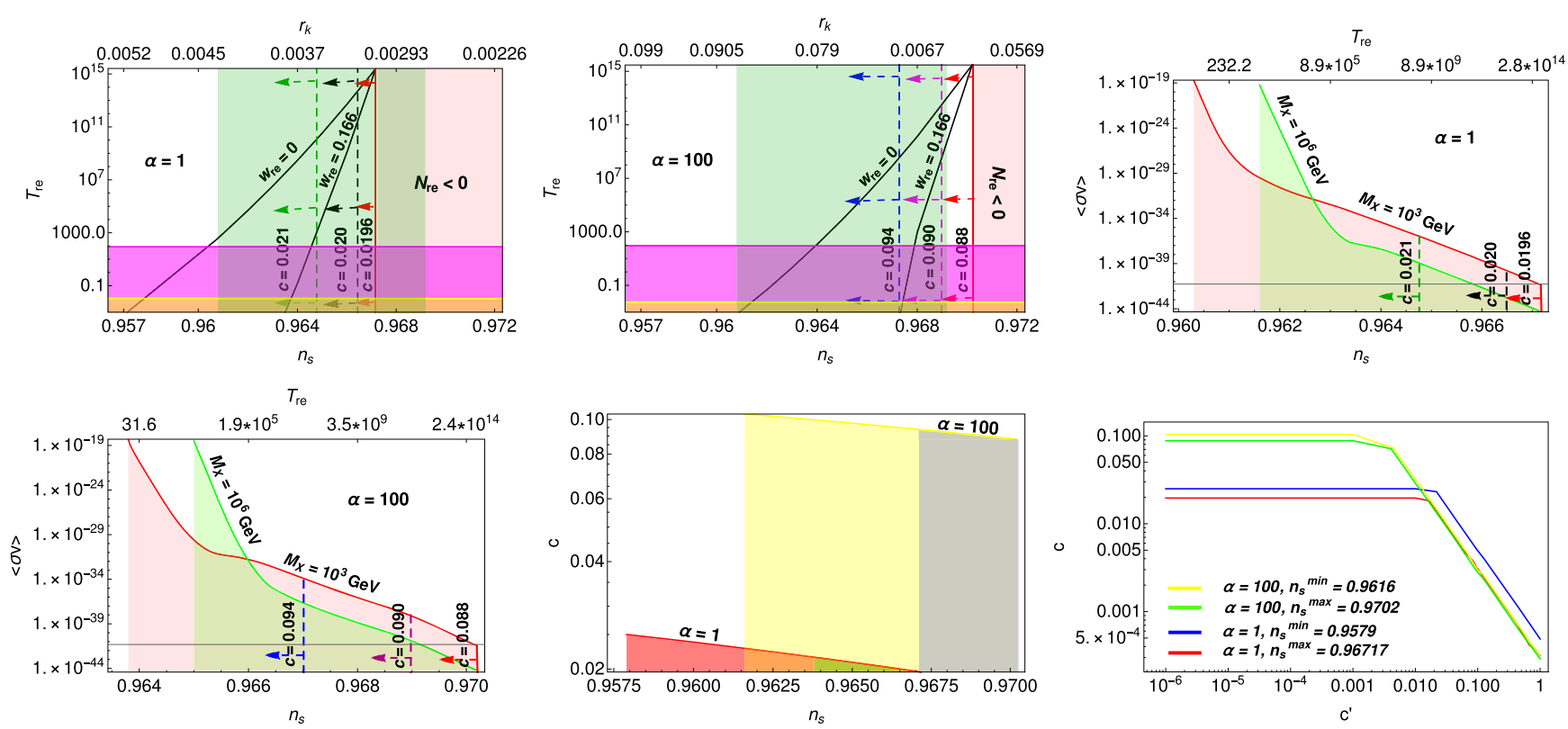

FIG. 3. All plots are the same as in Fig. 1. The main difference is that here we have plotted for the $\alpha$-attractor inflation model for $\alpha=(1,100)$. In the lower middle plot, the region under the yellow solid line is for $\left(\omega_{r e}, \alpha\right)=(0,100)$ and the dark blue region is for $\left(\omega_{r e}, \alpha\right)=\left(\frac{1}{6}, 100\right)$. In the same plot, the region under the red solid line is for $\left(\omega_{r e}, \alpha\right)=(0,1)$ and the dark green region is for $\left(\omega_{r e}, \alpha\right)=\left(\frac{1}{6}, 1\right)$.

where

$$
\begin{aligned}
N_{k} & =\frac{3 \alpha}{4 n}\left[e^{\sqrt{\frac{2}{3 \alpha} \phi_{k}}}-e^{\sqrt{\frac{2}{3 \alpha} \phi_{\text {end }}}}-\sqrt{\frac{2}{3 \alpha}} \frac{\left(\phi_{k}-\phi_{\text {end }}\right)}{M_{p}}\right], \\
r_{k} & =\frac{64 n^{2}}{3 \alpha\left(e^{\sqrt{\frac{2}{3 \alpha} \phi_{k}}}-1\right)^{2}} .
\end{aligned}
$$

Similar to the previous inflation models, here also we have $c^{t \text { max }}$ corresponding to the instantaneous reheating, which is independent of the equation of state but dependent on $\alpha$. For example, we have $c^{t \max }=(0.0196,0.088)$ for $\alpha=(1,100)$. Further, the minimum value of the scalar spectral index corresponds to a maximum value of $c=c^{\text {max }}$, which again translates into a minimum value of the reheating temperature. This observation may naturally provide a constraint on the possible ultraviolet completion. Nonetheless, for a fixed value of $\alpha=1$, the aforementioned maximum values turn out to be $c^{\max }=$ $(0.025,0.0216)$ for effective equation of state $\omega_{r e}=\left(0, \frac{1}{6}\right)$. By using the above Eq. (32), it turns out that for any particular values of $c$ within $c^{t \max }<c<c^{\max }$ and $\alpha$, the maximum allowed value of the reheating temperature is different for different reheating equation of state $\omega_{r e}$. For example assuming the following parameter $(\alpha, c)=$ $(1,0.02)$, the maximum allowed values of the reheating temperature assume $6.9 \times 10^{13} \mathrm{GeV}$ and $5.3 \times 10^{11} \mathrm{GeV}$ for $\omega_{r e}=\left(0, \frac{1}{6}\right)$ respectively. In the same way, the maximum and minimum value of the reheating temperature from observation imposes a restriction on the allowed values of $c$. After combining Eqs. (32) and (33) one can find

$$
\left(c c^{\prime}\right)^{2} \leq \frac{128 n^{4}}{27 \alpha^{3}} \frac{\left(e^{\sqrt{\frac{2}{3 \alpha}} \frac{\phi}{M_{p}}}-2 n\right)^{2}}{\left(e^{\sqrt{\frac{2}{3 \alpha}} \frac{\phi}{M_{p}}}-1\right)^{6}} .
$$

By using the above equation we are able to find out the resultant allowed parameter space of $c$ and $c^{\prime}$ as shown in the last plot of Fig. 3.

At a fixed values of $M_{X}=10^{3} \mathrm{GeV}$ and $\alpha=1$, the lower limit of the annihilation cross section $\langle\sigma v\rangle^{\text {lower }}$ is restricted by $c$, for instance, $\langle\sigma v\rangle^{\text {lower }} \simeq 4.2 \times 10^{-42}$ for $c=0.0196$. So swampland conjecture constant $c$ is restrained on the annihilation cross section $\langle\sigma v\rangle$ (Fig. 3) the same way as shown in the chaotic and natural inflation model, the only difference is that here the $c$ values are quite lower. The lower limit of the dark-matter annihilation cross section starts increasing with increasing $c$, where we consider $c$ with in $c^{\max }>c>c^{t \max }$. As an example, $\langle\sigma v\rangle^{\text {lower }} \simeq\left(1.9 \times 10^{-40}, 1.4 \times 10^{-36}\right) \mathrm{GeV}^{-2}$ for $c=(0.020,0.021)$.

For the $\alpha$-attractor model, if we consider the reheating equation of state $\omega_{r e}=0$, the minimum value of $\Delta$ is found to be constrained within $\Delta^{\mathrm{min}}=((1.14-1.15),(4.8-5.3))$ for $\alpha=(1,100)$ respectively. As has already been discussed for the chaotic case, for the axion inflation, if we take the sample value of $\Delta=1.15$ within the aforementioned range, the maximum reheating temperature assumes 
$T_{r e}^{\max }=\left(5 \times 10^{12}, 1.2 \times 10^{9}\right) \mathrm{GeV}$ for $\omega_{r e}=(0,1 / 6)$. At fixed values of $M_{X}=10^{3} \mathrm{GeV}$ and $\alpha=1$, the lower limit of the dark matter annihilation cross section $\langle\sigma v\rangle^{\text {lower }} \simeq$ $\left(3.3 \times 10^{-37}, 2.2 \times 10^{-39}, 1.1 \times 10^{-41}\right) \mathrm{GeV}^{-2}$ for $\Delta=$ $(1.150,1.152,1.153)$. The lower limit of annihilation cross section begins decreasing with increasing $\Delta$, so the allowed range of $\langle\sigma v\rangle$ becomes wider.

\section{Supergravity inspired minimal plateau model [72]}

In this section we will consider a special class of supergravity inspired inflation potential,

$$
V_{S G}(\phi)=\frac{m^{4-n} \phi^{n}}{e^{-\frac{\phi^{2}}{2 M_{p}^{2}}}+\left(\frac{\phi}{\phi_{*}}\right)^{n}} .
$$

The shape of the potential depends on the mass scale $\phi_{*}$ and all other parameters are the same as in the previous inflationary model. One of the striking features of this model is that unlike axion and $\alpha$-attractor models, it fits well within PLANCK data for all possible values of $\phi_{*}$ from super- to sub-Planckian value. Our initial motivation was to figure out if with increasing $\phi_{*}$ the value of $\left(c, c^{\prime}\right)$ increases towards unity. However, we did not find such solutions. Nonetheless, for our numerical computation, we have chosen two values of $\phi_{*}=(0.05,0.1) M_{p}$. At the point of instant reheating $N_{r e} \simeq 0$, maximum scalar spectral index assumes $n_{s}^{\max } \simeq(0.9722,0.9698)$ for the aforementioned two values of $\phi_{*}=(0.05,0.1) M_{p}$ with effective equation of state, $\omega_{r e}=0$. Using the swampland conjecture the condition between the potential $V_{S G}(\phi)$ and the spectral index $n_{s}$ (also reheating temperature) satisfies the following inequalities:

$$
\begin{gathered}
M_{p} \frac{V_{S G}^{\prime}}{V_{S G}}=\frac{2 M_{p}^{2} \phi_{*}^{2}+\phi_{*}^{2} \phi^{2}}{M_{p} \phi_{*}^{2} \phi+e^{\frac{\phi^{2}}{2 M_{p}^{2}}} M_{p} \phi^{3}} \geq c, \\
M_{p}^{2} \frac{V_{S G}^{\prime \prime}}{V_{S G}} \leq-c^{\prime},
\end{gathered}
$$

where

$$
M_{p}^{2} \frac{V_{S G}^{\prime \prime}}{V_{S G}}=\frac{\phi_{*}^{2}\left(2 M_{p}^{4}\left(\phi_{*}^{2}-3 e^{\frac{\phi^{2}}{2 M_{p}^{2}}} \phi^{2}\right)+M_{p}^{2} \phi^{2}\left(5 \phi_{*}^{2}-3 e^{\frac{\phi^{2}}{2 M_{p}^{2}}} \phi^{2}\right)+\phi^{4}\left(\phi_{*}^{2}-e^{\frac{\phi^{2}}{2 M_{p}^{2}}} \phi^{2}\right)\right)}{M_{p}^{2} \phi^{2}\left(\phi_{*}^{2}+e^{\frac{\phi^{2}}{2 M_{p}^{2}}} \phi^{2}\right)^{2}} .
$$

Similarly, the constraints on $\Delta$ will be obtained from distance conjecture Eq. (3) which transforms into

$$
\frac{\Delta \phi}{M_{p}}=\frac{r_{k}^{\frac{1}{2}} N_{k}}{2 \sqrt{2}} \leq \Delta,
$$

where

$$
\begin{aligned}
r_{k} & =\frac{8 \phi_{*}^{4}\left(2 M_{p}^{2}+\phi^{2}\right)^{2}}{M_{p}^{2} \phi^{2}\left(\phi_{*}^{2}+e^{\frac{\phi^{2}}{2 M_{p}^{2}}} \phi^{2}\right)}, \\
N_{k} & =\int_{\phi_{k}}^{\phi_{\text {end }}} \frac{\phi\left(\phi_{*}^{2}+\exp \left[\phi^{2} /\left(2 M_{p}^{2}\right)\right] \phi^{2}\right)}{\phi_{*}^{2}\left(2 M_{p}^{2}+\phi^{2}\right)}|d \phi| .
\end{aligned}
$$

From this point onward let us consider some very specific points about the model and also mention some numerical estimates as we have already done for all the previous cases. At the point of instant reheating which is defined as $N_{r e}=0$, the $c$ parameter assumes very specific values identified as $c^{t \text { max }}$. For our present supergravity inspired model, $c^{t \max }=0.005$ for $\phi_{*}=0.1 M_{p}$ and it decreases with $\phi_{*}$ very slowly. Although maximum values of $c\left(c^{\max }\right)$ are the same for different values of equation of state but it changes with different $\phi^{*}$ values. Those values turn out to be $c^{\max }=(0.0066,0.0058)$ for $\phi_{*}=$ $(0.05,0.1) M_{p}$ respectively. Similar to other models discussed above, any particular value of $c$ between $c^{\max }>$ $c>c^{t \text { max }}$ provides an upper limit on the reheating temperature for a given value of $\phi_{*}$ and $\omega_{r e}$ as shown in the first and second plots of Fig. 4. For example, maximum allowed values of the reheating temperature turn out to be $T_{r e}^{\max }=$ $\left(1.1 \times 10^{13}, 1.3 \times 10^{10}\right) \mathrm{GeV}$ for two different effective equations of state $\omega_{r e}=(0,1 / 6)$ considering fixed values of $\left(\phi_{*}, c\right)=\left(0.1 M_{p}, 0.0055\right)$. Inversely, we can say that the observational limit of reheating temperature imposes a restriction on the allowed free parameter space of the swampland conjecture $\left(c, c^{\prime}\right)$. In order to explain that, as has been done for the previous models, one combines Eqs. (39) and (41), and the inequality turns out to be

$\left(c c^{\prime}\right)^{2} \leq \frac{\phi_{*}^{8}\left(2 M_{p}^{2}+\phi^{2}\right)^{2}\left(2 M_{p}^{4}\left(\phi_{*}^{2}-3 e^{\frac{\phi^{2}}{2 M_{p}^{2}}} \phi^{2}\right)+M_{p}^{2} \phi^{2}\left(5 \phi_{*}^{2}-3 e^{\frac{\phi^{2}}{2 M_{p}^{2}}} \phi^{2}\right)\right)^{2}}{M_{p}^{6} \phi^{6}\left(\phi_{*}^{2}+e^{\frac{\phi^{2}}{2 M_{p}^{2}}} \phi^{2}\right)^{6}}+\frac{\phi_{*}^{8}\left(2 M_{p}^{2}+\phi^{2}\right)^{2}\left(\phi^{4}\left(\phi_{*}^{2}-e^{\frac{\phi^{2}}{2 M_{p}^{2}}} \phi^{2}\right)\right)^{2}}{M_{p}^{6} \phi^{6}\left(\phi_{*}^{2}+e^{\frac{\phi^{2}}{2 M_{p}^{2}}} \phi^{2}\right)^{6}}$. 

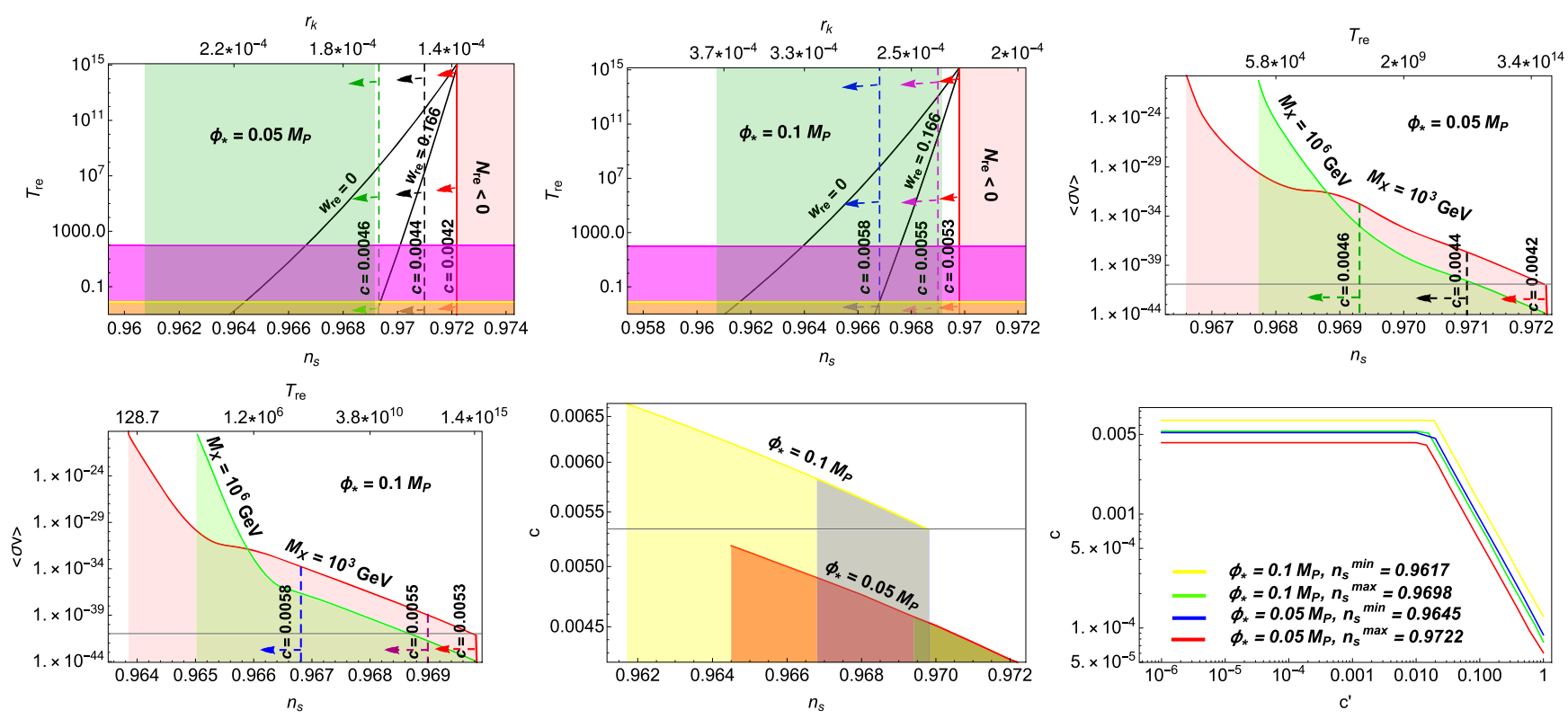

FIG. 4. All plots are the same as in Fig. 1. The main difference is that here we have plotted for supergravity inflation model for $\phi_{*}=(0.05,0.1) M_{p}$. In the lower middle plot, the region under the yellow solid line is for $\left(\omega_{r e}, \phi_{*}\right)=\left(0,0.1 M_{p}\right)$ and the dark blue region is for $\left(\omega_{r e}, \phi_{*}\right)=\left(\frac{1}{6}, 0.1 M_{p}\right)$. In the same plot, the region under the red solid line is for $\left(\omega_{r e}, \phi_{*}\right)=\left(0,0.1 M_{p}\right)$ and the dark green region is for $\left(\omega_{r e}, \phi_{*}\right)=\left(\frac{1}{6}, 0.1 M_{p}\right)$.

The resulting constraints on $\left(c, c^{\prime}\right)$ are displayed in the last plot of Fig. 4.

Now we will discuss the effect of the swampland conjecture on the dark-matter parameter space. As already mentioned for the previous inflationary models, the lower limit of the annihilation cross section is modified by different values of $c$ once we fixed the dark-matter mass $M_{X}$ and mass scale of the potential $\phi_{*}$. For $M_{X}=10^{3} \mathrm{GeV}$ and $\quad \phi_{*}=0.1 M_{p}, \quad\langle\sigma v\rangle^{\text {lower }} \simeq\left(8 \times 10^{-42}, 1 \times 10^{-39}\right.$, $\left.1.9 \times 10^{-34}\right) \mathrm{GeV}^{-2}$ for $c=(0.0053,0.0055,0.0058)$.
The annihilation cross section of the dark-matter becomes more restricted with increasing $c$ within $c^{\max }>c>c^{t \max }$.

For the present scenario the allowed range of minimum values $\Delta$ turns out to be $\Delta^{\mathrm{min}}=((0.2976-0.3078)$, $(0.2315-0.2436))$ for $\phi_{*}=(0.1,0.05) M_{p}$ as shown in Fig. 5. As has been discussed for the other models, if we chose a particular value of $\Delta=0.3077$ within the aforementioned range, one obtains $T_{r e}^{\max }=\left(1.1 \times 10^{13}\right.$, $\left.1.3 \times 10^{10}\right) \mathrm{GeV}$ for $\omega_{r e}=\left(0, \frac{1}{6}\right)$ and $\langle\sigma v\rangle^{\text {lower }} \simeq 1 \times$ $10^{-39} \mathrm{GeV}^{-2}$ with $M_{X}=10^{3} \mathrm{GeV}$ and $\phi_{*}=0.1 M_{p}$.

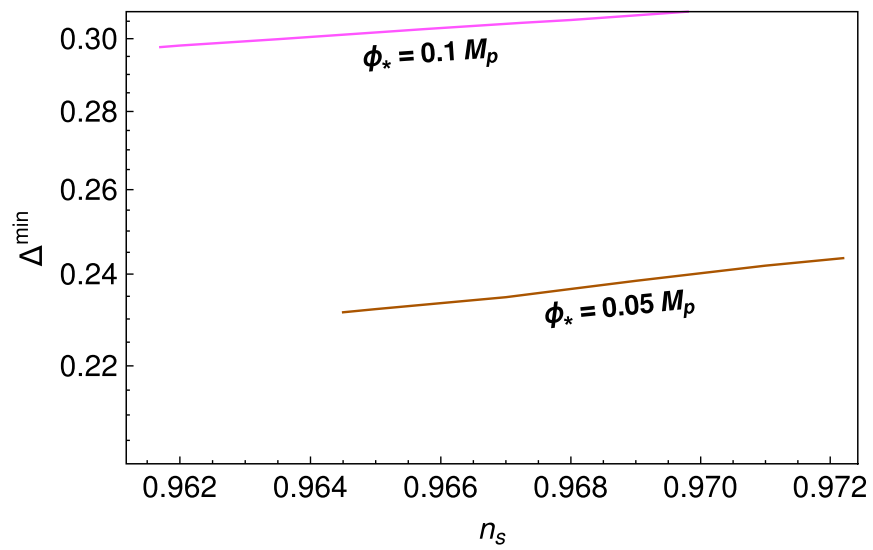

FIG. 5. We plot the variation of minimum values of $\Delta\left(\Delta^{\mathrm{min}}\right)$ as a function of $n_{s}$ within maximum and minimum values of reheating temperature with effective equation of state $\omega_{r e}=0$. In the first plot, the red solid line is used for chaotic inflation model with $n=2$, black and green lines are for natural inflation with $f=(50,10) M_{p}$, blue and yellow lines indicate the $\alpha$-attractor model for $\alpha=(1,100)$. In the second plot, the brown and pink lines are for the supergravity inspired minimal plateau model with $\phi_{*}=(0.05,0.1) M_{p}$. 


\section{SUMMARY AND DISCUSSION}

Swampland conjecture has gained significant attention mainly because of its potential to validate or invalidate a large class of low energy effective theories proposed in diverse physics problems. It mainly deals with a scalar field and its possible nature of the potential which is conjectured to follow certain constraints. Three parameters $\left(c, c^{\prime}\right.$ and $\left.\Delta\right)$ are conjectured to be of the order unity such that the field theory under consideration can have consistent ultraviolet completion when minimally coupled with gravity. One of the best candidate scalar fields is inflaton which has been proven to be successful in explaining a large volume of cosmological observations. However, it turned out that a more successful inflation scenario is more incompatible with the swampland conjecture it becomes. In the present paper instead of taking swampland parameters to be of order unity, we considered them free and analyze their impact on the other cosmological parameters with special emphasis on the reheating phase. Based on our analysis so far let us try to point out the main outcomes. We have considered four different types of inflaton potential and studied the consequence of swampland conjectures on those and constrain the parameter space. However, we must say that the conventional slow roll potential is very much constrained by the conjecture. For all the models under consideration, what we found is that the possible values of $c$ are always less than unity. The maximum possible value one could get $c \simeq 10^{-1}$ is for chaotic inflation which predicts a higher value of tensor to scalar ratio. Moreover, we studied other models, such as axion, $\alpha$-attractor, supergravity inspired inflation, which are either marginally or fully consistent with PLANCK data, and the maximum possible value of $c$ turns out to be even smaller for those models. This result is intimately connected with the prediction of smaller value of the tensor to scalar ratio $r$. However, this has already been observed before.

Interestingly, the swampland distance conjecture parameter $\Delta$ appeared to be consistent with some of the inflation models, we considered. As mentioned earlier, this conjecture has an obvious connection with the well-known Lyth bound in the inflationary context, $\Delta>1$ generically corresponds to super-Planckian inflation which is known to be inconsistent in the effective theory framework. Interestingly as expected, $\Delta$ turned out to be larger than unity for chaotic and natural inflation model. On the other hand, for the $\alpha$-attractor model with $\alpha=1$, we find $\Delta^{\text {min }}$ is around order 1 . For a supergravity inspired minimal plateau model $\Delta^{\min }$ is even smaller than unity. Therefore, even though in terms of $c$ parameter, all the inflationary models under consideration seemed to be inconsistent with the refined swampland conjecture, $\alpha$-attractor and supergravity inspired models can be consistent with the distance conjecture. In any case, our focus in this paper was more on the impact of those inflationary constraints of $\left(c, c^{\prime}\right.$ and $\left.\Delta\right)$ on the reheating phase and dark matter phenomenology. In Figs. 1-4 we have considered different cosmological parameters such as $\left(n_{s}, T_{r e},\langle\sigma v\rangle\right)$, and studied their interdependence and constraints from the swampland conjecture. In the usual reheating constraint analysis, the reheating temperature varies widely within the $2 \sigma$ range of $n_{s}$, and consequently so does the dark matter cross section $\langle\sigma v\rangle$ for a given dark matter mass. Since swampland conjecture is an inequality, for the model under consideration, parameters of swampland conjecture $c$ and $\Delta$ both individually provide an upper bound on $T_{r e}$ and lower bound on $\langle\sigma v\rangle$ for a given dark matter mass $M_{X}$. However, since reheating temperature is already constrained by the BBN, for all the models we have an associated maximum value of swampland parameter $c^{\max }$ as shown in Table I, where we considered a natural value of the effective equation of state $\omega_{r e}=0$. Additionally in Fig. 5 we have plotted the allowed range of $\Delta^{\mathrm{min}}$ arising from the minimum and maximum values of the reheating temperature associated with BBN at temperature $T \sim 10^{-2} \mathrm{GeV}$ and $N_{r e} \sim 0$, respectively.

Considering a different class of inflationary models, one of our important observations is the existence of maximum possible reheating temperature $T_{r e}^{\max } \simeq 10^{15} \mathrm{GeV}$ irrespective of the models. Importantly, however, the associated swampland parameter $c=c^{t \max }$ and $\Delta$ are depending upon the models and their parameters. Another important observation from our analysis is that for each value of $c$ within $\left(c^{\max }, c^{t \max }\right)$ and $\Delta$ within the allowed range, there exists an associated maximum possible value of reheating temperature $T_{r e}^{\max }(c, \Delta)$ and consequently minimum value of $\langle\sigma v\rangle^{\operatorname{lower}}(c, \Delta)$ for fixed dark matter mass. For example, the $\alpha$-attractor model predicts $T_{r e}^{\max }(c, \Delta)=5 \times 10^{12} \mathrm{GeV}$ and $\langle\sigma v\rangle^{\text {lower }}(c, \Delta) \simeq 2.2 \times 10^{-39} \mathrm{GeV}^{-2}$ for $(c, \Delta)=(0.0203$, $1.152)$ with $\left(\alpha, M_{X}\right)=\left(1,10^{3} M_{p}\right)$, which is the wellknown Higgs/Starobinsky inflation model. Interestingly for our supergravity inspired minimal model which agrees very well with the PLANCK data at all values of $\phi_{*}$ turned out to set maximum possible values of $c \simeq 10^{-2}$ and minimum possible values of $\Delta^{\mathrm{min}} \simeq 10^{-1}$. For all the

TABLE I. Models and their associated $c^{\max }$ value.

\begin{tabular}{|c|c|c|c|c|c|c|c|}
\hline & \multirow[b]{2}{*}{ Chaotic } & \multicolumn{2}{|c|}{$\alpha$-attractor } & \multicolumn{2}{|c|}{ Axion } & \multicolumn{2}{|c|}{ Supergravity } \\
\hline & & $\alpha=1$ & $\alpha=100$ & $f=10 M_{p}$ & $f=50 M_{p}$ & $\phi_{*}=0.05 M_{p}$ & $\phi_{*}=0.1 M_{p}$ \\
\hline$c^{\max }$ & 0.1448 & 0.025 & 0.0104 & 0.128 & 0.144 & 0.0052 & 0.0053 \\
\hline
\end{tabular}


models under consideration, the value of $c$ turned out to be bounded within $\left(0, c^{\max }\right)$, and that of $c^{\prime}$ is within $(0,1)$. Finally, if we consider all forms of the swampland conjectures to be true with their theoretical parameter values, namely $c \gtrsim 1$ and $\Delta \lesssim 1$, our supergravity inspired model turned out to be the only model consistent with the distance conjecture. All models are strongly disfavored in the realm of the refined swampland conjecture.
[1] H. Ooguri, E. Palti, G. Shiu, and C. Vafa, Phys. Lett. B 788, 180 (2019).

[2] D. H. Lyth, Phys. Rev. Lett. 78, 1861 (1997).

[3] H. Ooguri and C. Vafa, Nucl. Phys. B766, 21 (2007).

[4] D. Klaewer and E. Palti, J. High Energy Phys. 01 (2017) 088.

[5] D. Andriot, Phys. Lett. B 785, 570 (2018).

[6] G. Dvali and C. Gomez, Fortschr. Phys. 67, 1800092 (2019).

[7] T. Bjorkmo, arXiv:1902.10529.

[8] A. Achcarro and G. A. Palma, J. Cosmol. Astropart. Phys. 02 (2019) 041.

[9] S. K. Garg and C. Krishnan, arXiv:1807.05193.

[10] C. Roupec and T. Wrase, Fortschr. Phys. 67, 1800082 (2019).

[11] J. L. Lehners, J. Cosmol. Astropart. Phys. 11 (2018) 001.

[12] F. Denef, A. Hebecker, and T. Wrase, Phys. Rev. D 98, 086004 (2018).

[13] E. Colgin, M. H. P. M. Van Putten, and H. Yavartanoo, Phys. Lett. B 793, 126 (2019).

[14] E. Palti, arXiv:1903.06239.

[15] J. H. Park, arXiv:1902.04559.

[16] T. Bjorkmo and M. C. D. Marsh, J. High Energy Phys. 04 (2019) 172.

[17] A. Kobakhidze, arXiv:1901.08137.

[18] D. Andriot, Fortschr. Phys. 67, 1800103 (2019).

[19] H. Matsui and F. Takahashi, Phys. Rev. D 99, 023533 (2019).

[20] M. Cicoli, S. De Alwis, A. Maharana, F. Muia, and F. Quevedo, Fortschr. Phys. 67, 1800079 (2019).

[21] W. H. Kinney, S. Vagnozzi, and L. Visinelli, arXiv:1808 .06424

[22] C. Damian and O. Loaiza-Brito, Fortschr. Phys. 67, 1800072 (2019).

[23] I. Ben-Dayan, arXiv:1808.01615.

[24] J. P. Conlon, Int. J. Mod. Phys. A 33, 1850178 (2018).

[25] K. Dasgupta, M. Emelin, E. McDonough, and R. Tatar, J. High Energy Phys. 01 (2019) 145.

[26] Y. Akrami, R. Kallosh, A. Linde, and V. Vardanyan, Fortschr. Phys. 67, 1800075 (2019).

[27] D. Andriot and C. Roupec, Fortschr. Phys. 67, 1800105 (2019).

[28] C. Han, S. Pi, and M. Sasaki, Phys. Lett. B 791, 314 (2019)

[29] J. Moritz, A. Retolaza, and A. Westphal, Fortschr. Phys. 67, 1800098 (2019).

[30] H. Murayama, M. Yamazaki, and T. T. Yanagida, J. High Energy Phys. 12 (2018) 032.

[31] M. C. D. Marsh, Phys. Lett. B 789, 639 (2019).
[32] S. Brahma and M. Wali Hossain, J. High Energy Phys. 03 (2019) 006.

[33] K. Choi, D. Chway, and C. S. Shin, J. High Energy Phys. 11 (2018) 142.

[34] S. Das, Phys. Rev. D 99, 083510 (2019).

[35] U. Danielsson, J. High Energy Phys. 04 (2019) 095.

[36] D. Wang, arXiv:1809.04854.

[37] L. Visinelli and S. Vagnozzi, Phys. Rev. D 99, 063517 (2019).

[38] I. Bena, E. Dudas, M. Graña, and S. Lüst, Fortschr. Phys. 67, 1800100 (2019).

[39] R. Brandenberger, R. R. Cuzinatto, J. Fröhlich, and R. Namba, J. Cosmol. Astropart. Phys. 02 (2019) 043.

[40] R. H. Brandenberger, arXiv:1809.04926.

[41] J. Quintin, R. H. Brandenberger, M. Gasperini, and G. Veneziano, Phys. Rev. D 98, 103519 (2018).

[42] L. Heisenberg, M. Bartelmann, R. Brandenberger, and A. Refregier, Fortschr. Phys. 67, 1800075 (2019).

[43] B. M. Gu and R. Brandenberger, arXiv:1808.03393.

[44] L. Heisenberg, M. Bartelmann, R. Brandenberger, and A. Refregier, Phys. Rev. D 98, 123502 (2018).

[45] K. Dimopoulos, Phys. Rev. D 98, 123516 (2018).

[46] J. Ellis, B. Nagaraj, D. V. Nanopoulos, and K. A. Olive, J. High Energy Phys. 11 (2018) 110.

[47] C. M. Lin, K. W. Ng, and K. Cheung, arXiv:1810.01644.

[48] K. Hamaguchi, M. Ibe, and T. Moroi, J. High Energy Phys. 12 (2018) 023.

[49] M. Kawasaki and V. Takhistov, Phys. Rev. D 98, 123514 (2018).

[50] M. Motaharfar, V. Kamali, and R. O. Ramos, Phys. Rev. D 99, 063513 (2019).

[51] S. D. Odintsov and V. K. Oikonomou, arXiv:1810.03575.

[52] A. Ashoorioon, Phys. Lett. B 790, 568 (2019).

[53] G. Obied, H. Ooguri, L. Spodyneiko, and C. Vafa, arXiv: 1806.08362 .

[54] J. Moritz, A. Retolaza, and A. Westphal, Phys. Rev. D 97, 046010 (2018).

[55] R. Kallosh, A. Linde, E. McDonough, and M. Scalisi, J. High Energy Phys. 03 (2019) 134; R. Kallosh, Phys. Rev. D 99, 066003 (2019); Y Hamada, A. Hebecker, G. Shiu, and P. Soler, arXiv:1902.01410; F. F. Gautason, V. Van Hemelryck, T. Van Riet, and G. Venken, arXiv:1902 .01415; R. Blumenhagen, D. Klaewer, and L. Schlechter, arXiv:1902.07724.

[56] A. Kehagias and A. Riotto, Fortschr. Phys. 66, 1800052 (2018).

[57] M. Scalisi and I. Valenzuela, arXiv:1812.07558.

[58] H. Fukuda, R. Saito, S. Shirai, and M. Yamazaki, Phys. Rev. D 99, 083520 (2019). 
[59] M. Raveri, W. Hu, and S. Sethi, Phys. Rev. D 99, 083518 (2019).

[60] V. Kamali, arXiv:1902.00701.

[61] M. Dias, J. Frazer, A. Retolaza, and A. Westphal, Fortschr. Phys. 67, 1800063 (2019).

[62] M. S. Seo, arXiv:1812.07670.

[63] L. Dai, M. Kamionkowski, and J. Wang, Phys. Rev. Lett. 113, 041302 (2014).

[64] J. L. Cook, E. Dimastrogiovanni, D. A. Easson, and L. M. Krauss, J. Cosmol. Astropart. Phys. 04 (2015) 047.

[65] D. Maity and P. Saha, Phys. Rev. D 98, 103525 (2018).

[66] G. F. Giudice, E. W. Kolb, and A. Riotto, Phys. Rev. D 64, 023508 (2001).
[67] A. D. Linde, Phys. Lett. 129B, 177 (1983).

[68] O. E. Núñez, J. Socorro, and R. Hernández-Jiménez (Planck Collaboration), Astrophys. Space Sci. 364, 69 (2019).

[69] K. Freese, J. A. Frieman, and A. V. Olinto, Phys. Rev. Lett. 65, 3233 (1990).

[70] R. Kallosh and A. Linde, J. Cosmol. Astropart. Phys. 07 (2013) 002; R. Kallosh, A. Linde, and D. Roest, J. High Energy Phys. 11 (2013) 198.

[71] M. Drewes, J. U. Kang, and U.R. Mun, J. High Energy Phys. 11 (2017) 072.

[72] D. Maity and P. Saha, Classical Quantum Gravity 36, 045010 (2019). 